\title{
Correction to: Sexual Behavior Prevalence and Its Predictors Among Students in an American University
}

\author{
Shayesteh Jahanfar ${ }^{1} \cdot$ Parvin $^{\text {Abedi }^{2}} \cdot$ Shahla Faal Siahkal ${ }^{3,4}$
}

Published online: 28 June 2021

(c) Springer Science+Business Media, LLC, part of Springer Nature 2021

\section{Correction to: Sexuality \& Culture https://doi.org/10.1007/s12119-021-09816-x}

The original version of this article unfortunately contained mistakes. It has been corrected in this correction.

1. In "Abstracts" section, on line 13, the "only age was" should read as "age and the level of education were"

2. In "Abstracts" section, on line 15, the score "OR: 0.09 CI 95\% [0.03, 0.16])" should read as "(RR: 0.09, CI 95\% [0.03, 0.16])"

3. In "Abstracts" section, on line 17, the score "(OR: 0.04, CI 95\% [0.71-0.17])" should read as "(RR: 0.04, CI 95\% [-0.17, 0.71])".

4. In "Abstracts" section, on line 19, at the end of sentence after years the score needs to be added "(RR: 0.09, CI 95\% [0.03, 0.16])"

5. In "Methodology" section, under the "Study Design and Sampling" heading, sample size in first line of the second paragraph should read as " $(n=924)$ "

The original article can be found online at https://doi.org/10.1007/s12119-021-09816-x.
Shahla Faal Siahkal
faalshahla1@yahoo.com
Shayesteh Jahanfar
shayesteh.jahanfar@tufts.edu
Parvin Abedi
parvinabedi@ymail.com

1 MPH Program, Department of Public Health and Community Medicine, Tufts University School of Medicine, Boston, USA

2 Midwifery Department, Menopause Andropause Research Center, Ahvaz Jundishapur University of Medical Sciences, Ahvaz, Iran

3 Department of Midwifery, Marand Branch, Islamic Azad University, Marand, Iran

4 Midwifery Department, Nursing and Midwifery School, Ahvaz Jundishapur University of Medical Sciences, Golestan Ave, Ahwaz, Iran 
Table 4 The unadjusted and adjusted RR of sexual behavior in association with sex education

\begin{tabular}{|c|c|c|}
\hline Sociodemographic factors & Unadjusted RR 95\%CI & Adjusted RR 95\%CI \\
\hline Age & $0.01(0.05,0.14)$ & $0.09(0.03,0.16)$ \\
\hline \multicolumn{3}{|l|}{ Gender } \\
\hline Male & $0.16(-0.63,0.94)$ & $0.27(-0.58,1.13)$ \\
\hline Female & 1 & 1 \\
\hline \multicolumn{3}{|l|}{ Education } \\
\hline Undergraduate & $-0.95(-2.59,-0.29)$ & $-0.46(-1.81,0.88)$ \\
\hline Graduate & 1 & 1 \\
\hline \multicolumn{3}{|l|}{ Income } \\
\hline$\$ 1000$ or less & $0.43(-0.45,1.31)$ & $-0.28(-1.81,0.69)$ \\
\hline More than $\$ 1000$ & 1 & 1 \\
\hline \multicolumn{3}{|l|}{ Religion } \\
\hline \multicolumn{3}{|l|}{ None } \\
\hline Catholic & $0.08(-0.29,0.44)$ & $0.03(-0.78,0.94)$ \\
\hline Attending religious event regularly & 1 & 1 \\
\hline \multicolumn{3}{|l|}{ Importance of religion } \\
\hline Not important & $-0.09(-0.51,0.32)$ & $-0.97(-0.24,0.21)$ \\
\hline Important & 1 & 1 \\
\hline \multicolumn{3}{|l|}{ Attending religious events regularly } \\
\hline Yes & $-0.52(-1.15,0.11)$ & $0.42(-1.95,0.05)$ \\
\hline No & 1 & 1 \\
\hline \multicolumn{3}{|l|}{ Sex education } \\
\hline Yes & $-0.38(-1.39,0.64)$ & $0.26(-0.91,0.66)$ \\
\hline No & 1 & 1 \\
\hline Score of STD knowledge & $0.08(-0.11,0.26)$ & $0.04(-0.17,0.71)$ \\
\hline
\end{tabular}

6. In "Result" section, under the "Sociodemographic Data" subheading, the $(n=256$, $31.8 \%)$ should be corrected as $(n=169,21.0 \%)$ on 5 th line and the "(were working $(n=520,68.8 \%))$," should be deleted on 6th line.

7. In "Result" section, under the "Relationship/Sexual Behavior" subheading, the following part need to be added after the last sentence.

"In total, $86.9 \%$ of students described their relationship were serious. In $95.4 \%$ of cases, their boy/girlfriend was single when they started the relationship and someone who was working or was a full-time student (93.8\%). It is worth noting that there are still about $11.6 \%$ of students who date while dating others simultaneously. Moreover, about $71 \%$ of students have shorter than two terms (10 months) relationships. They end their relationship one-sidedly without agreement from both parties (79.2\%). Many students consider relationships as experimental, as $13.1 \%$ think of their relationship as casual. Also, $60.4 \%$ of students reported that their relationship lasted more than 10 months, and in $79.2 \%$ of cases, one-side decided to end the relationship." 
8. In "Result" section, under the "Regression Analysis" subheading, remove (=) in the scores. The corrected score should read as "(RR: 0.09, CI 95\% [0.03, 0.16])"

9. In "Discussion" section, the sentences from "In total, $86.9 \%$ of students described their relationship decided to end the relationship" should be removed.

10. In "Discussion" section, the "only age was" should be corrected as "age and the level of education were"

11. The correct version of "Table 4" is given below.

12. In "Discussion" section under the "Casual or Serious Relationship" heading, delete "becoming" in 2nd line.

13. In "Discussion" section under the "Regret having Intercourse with Boy/girlfriend for the First Time "heading, citation should be given for "(Wright 2010)" reference.

14. In "Discussion" section under the "Predictors of Relationship/Sexual Behaviour", "education" in 1st line should be corrected as "sex education".

In "Conclusion" section, "only age was" in 6th line should be corrected as "age and the level of education were"

Publisher's Note Springer Nature remains neutral with regard to jurisdictional claims in published maps and institutional affiliations. 